\title{
A Digitally Programmable Temperature Controller Based on a Phase-Locked Loop
}

\author{
OSAMA I. MOHAMED, STUDENT MEMBER, IEEE, SERGIO SHOJI, \\ AND KENZO WATANABE, SENIOR MEMBER, IEEE
}

\begin{abstract}
A digitally programmable temperature controller is developed based on a phase-locked loop (PLL). Temperature under control is converted to frequency form and then compared, in a phase detector, with the reference frequency which corresponds to the target temperature. The detected phase drives the actuator. The frequency difference is also detected by a one-chip microcomputer which changes the reference frequency according to a proportional-integral-derivative operation. A prototype controller achieved $0.1^{\circ} \mathrm{C}$ stability in the temperature control of a water bath.
\end{abstract}

\section{INTRODUCTION}

$\mathrm{C}$ THEMICAL and physical reactions are highly sensitive to temperature and thus temperature control is indispensable for industrial processes. To that aim, several controllers have been developed which use computers as the central unit [1]-[4]. By virtue of their powerful computing engines these controllers feature high accuracy, programmability, and adaptability. The temperature sensors used in these systems, however, provide their outputs in current or voltage form. Therefore, a high precision analog-to-digital converter is required for interfacing the sensor with the central unit. In addition, some controllers require digital-to-analog converters to drive the actuator according to the control algorithm.

Intensive effort is now devoted to the development of an intelligent sensor that provides the sensed variable in digital form [5]. A variety of output formats are possible, but frequency encoding requires only a simple interface and provides the best method of transmitting data. In view of this trend, a temperature control method compatible with such an intelligent sensor has been developed.

Section II describes the principles of operation and the architecture. To prevent an oscillatory response due to the slow heat transfer process, a proportional-integral-derivative (PID) control is essential. Its algorithm is also given. An integral part of the architecture is the temperature-tofrequency $(T / F)$ converter. Section III describes a prototype controller using a newly developed $T / F$ converter. Experimental results are presented which confirm the control principles. The paper concludes (Section IV) with a description of the potential applications and future works.

\footnotetext{
Manuscript received April 20,1988. This work was supported by the Kurabe Industrial Company, Kamimura, Japan.

The authors are with the Research Institute of Electronics, Shizuoka University, Hamamatsu, 432 Japan.

IEEE Log Number 8823504.
}

\section{ARChitecture}

\section{A. Hardware}

The block diagram of the temperature controller is shown in Fig. 1. It can be divided into two main blocks: a one-chip microcomputer as the central unit, and the phase-locked loop (PLL) consisting of the $T / F$ converter, frequency divider, phase detector, and power stage.

The $T / F$ converter corresponds to a voltage-controlled oscillator in a conventional PLL. For simplicity, its output frequency $f_{T}$ is assumed to be proportional to the temperature $T$ under control,

$$
f_{T}=f_{o}+S_{c} T
$$

where $f_{o}$ is the offset frequency and $S_{c}$ is the conversion sensitivity. Comparing the two frequencies, $f_{T}$ and $f_{r} / D$, where $f_{r}$ is the oscillation frequency of the stable clock generator and $D$ is the divisor set to the frequency divider, the phase detector produces the error signal to drive the power stage. The transfer function of the PLL is expressed as

$$
H_{p}(s)=\frac{\omega_{n}^{2}}{S^{2}+2 \zeta \omega_{n} S+\omega_{n}^{2}}
$$

where the damping factor $\zeta$ and the natural frequency $\omega_{n}$ are given, respectively, by

$$
\begin{aligned}
\zeta & =\frac{1}{2 R} \sqrt{\frac{1}{C S_{c} S_{d} G}} \\
\omega_{n} & =\sqrt{\frac{S_{c} S_{d} G}{C}}
\end{aligned}
$$

where $S_{d}$ is the sensitivity of the phase detector, $G$ is the transfer gain of the power stage, $C$ is the thermal capacity of the liquid under temperature control, and $R$ is the thermal resistance to heat flow through the tank surface. It is clear from (2) that if $D$, given by

$$
D=\frac{f_{r}}{f_{o}+S_{c} T_{s}}
$$

where $T_{s}$ is the target temperature, is set to the frequency divider, then the PLL controls temperature $T$ to track $T_{s}$.

The damping factor is usually less than 1 and thus the overshoot and undershoot appear in the step response of 


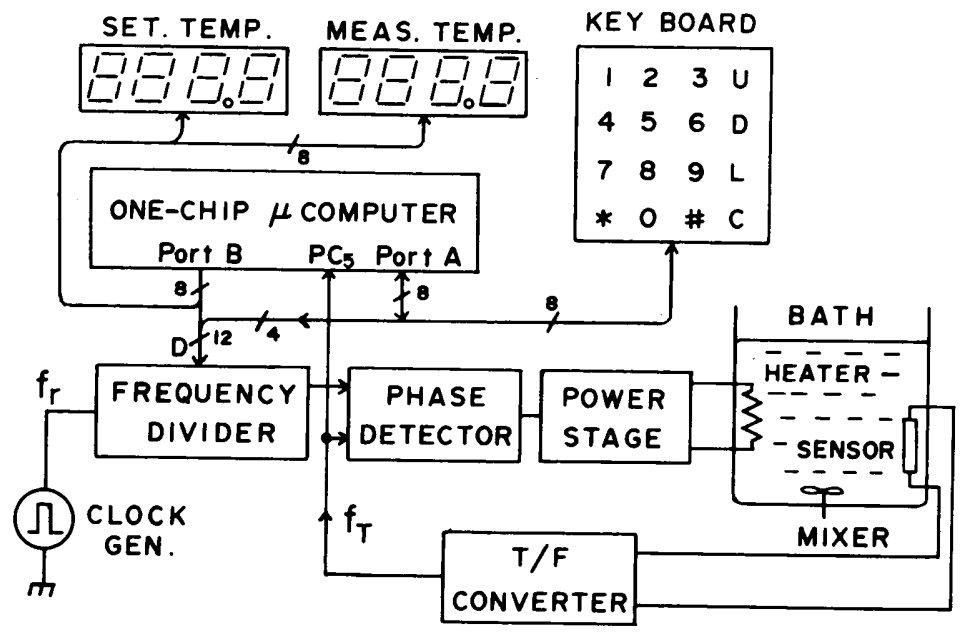

Fig. 1. The block diagram of the temperature controller.

PLL. For avoiding such an oscillation, and obtaining quick and stable response, the PID control is indispensable. To this aim, a one-chip microcomputer measures $f_{T}$ and compares it with $f_{s}$ to calculate the divisor $D$ appropriate for the PID control. Its details are described below.

\section{B. Software}

The control software assumes an 8-bit, one-chip microcomputer $\mu$ PD-7811 which accommodates, besides CPU, ROM, and RAM, three parallel I/O ports, two 8-bit timers, and a 16-bit event counter. The I/O port assignment is as follows: Port $A$ is assigned to the keyboard for inputting the target temperature. Port $B$ and the upper half of port $A$ are used for setting the divisor $D$ for the 12-bit frequency divider. Port B is also used for dynamic display of the target temperature $T_{s}$ and temperature $T$ under control, which is found by counting the output of the $T / F$ converter applied to the fifth bit $\mathrm{PC}_{5}$ of port $\mathrm{C}$. The other bits of port $\mathrm{C}$ are used to issue timing scales to latch and display dynamically, $T_{s}$ and $T$, and to set the divisor $D$ for the frequency divider.

One of the two timers, timer $A$, is used for generating an interrupt request signal every $1 /(30 \times 8) \mathrm{s}$ to scan the dynamic display and the keyboard sequentially. The other timer, timer $B$, is connected in parallel with timer $A$ to produce a gate signal every $0.5 \mathrm{~s}$ during which the event counter counts the output of the $T / F$ converter to measure $f_{T}$.

Fig. 2 shows the flowchart of the main program developed with the above-mentioned hardware architecture in mind. The program first initializes all registers, timers, and the event counter, and then waits for the interrupt signals from the timers. Upon receiving the interrupt request from timer $A$, the program scans the keyboard to input the target temperature and also issues the scanning signal for the dynamic display of $T_{s}$ and $T$. The interrupt request from timer $B$, on the other hand, commands the event counter to measure $f_{T}$. Using $f_{T}$ thus measured and the target temperature equivalent frequency $f_{s}$, the program executes the PID algorithm according to the flowchart shown in Fig. 3.

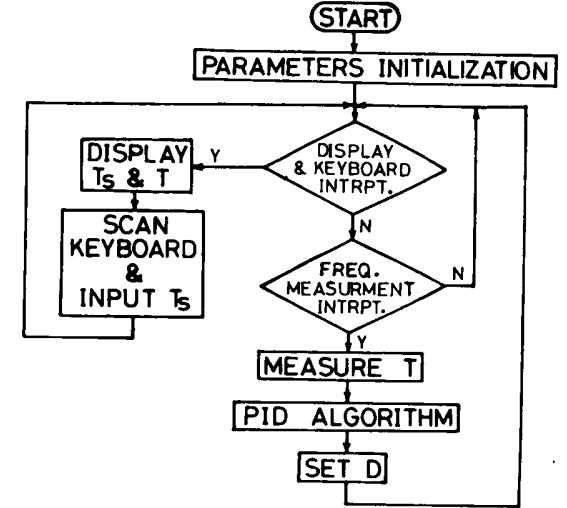

Fig. 2. The flowchart of the main program.

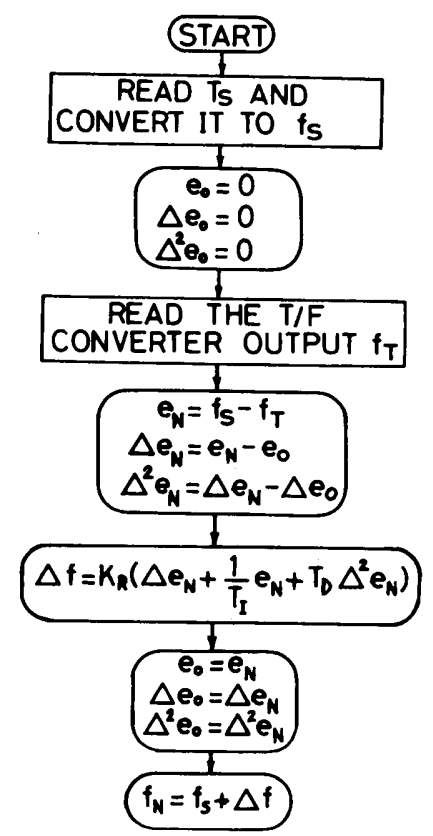

Fig. 3. The flowchart of the PID algorithm.

The PID algorithm can be expressed as

$$
f(t)=K_{R}\left\{e(t)+\frac{1}{T_{i}} \int e(t) d t+T_{d} \frac{d e(t)}{d t}\right\}
$$

where $f(t)$ is the control parameter, $e(t)$ is the difference between $f_{T}$ and $f_{s}, K_{R}$ is the gain, and $T_{i}$ and $T_{d}$ are the integration and derivation constants, respectively.

Expressing (6) in the difference equation form, we have

$$
\Delta f=K_{R}\left(\Delta e_{n}+\frac{1}{T_{i}} e_{n}+T_{d} \Delta^{2} e_{n}\right)
$$

where $e_{n}$ is the error between $f_{T}$ and $f_{s}$, and $\Delta e_{n}$ is the difference between the new and old errors. The PID program calculates $\Delta f$ and recognizes that the frequency $f_{n}$ with which $f_{T}$ is compared in the phase detector is $f_{s}+$ $\Delta f$. Updating $D$ depending on this operation, the program waits again for an interrupt signal.

\section{EXPERIMENTAL REsUlts}

To confirm the principles of operation, a prototype controller was built using off-the-shelf components except the $T / F$ converter. The clock generator used is a $12-\mathrm{MHz}$ 


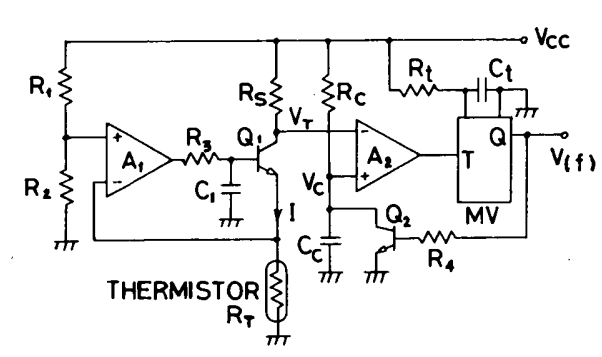

Fig. 4. The circuit diagram of the $T / F$ converter.

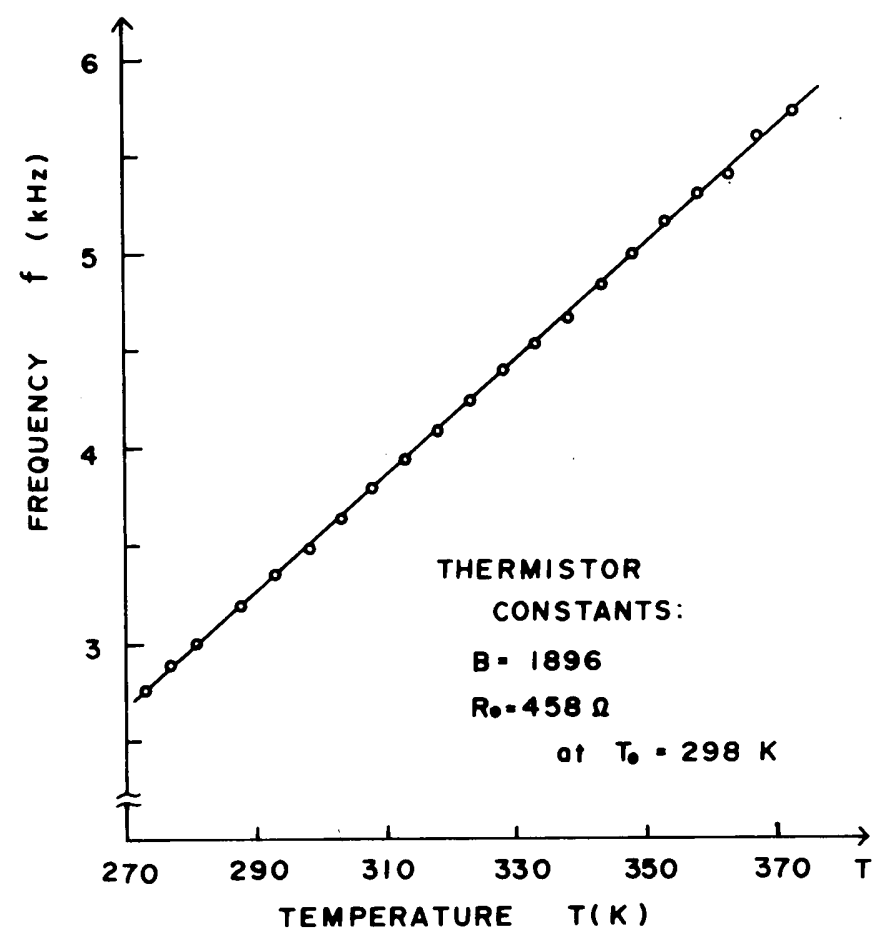

Fig. 5. The oscillation frequency $f$ versus temperature $T$ measured by a prototype $T / F$ converter.

crystal oscillator. The power stage is the solid-state relay of zero-crossing detection which switches $100-\mathrm{V}$ ac on and off depending on the output of the CMOS phase detector.

For temperature control up to $100^{\circ} \mathrm{C}$, a $T / F$ converter using a thermistor is developed [6]. Its circuit diagram is shown in Fig. 4. Op-amp $A_{1}$ and transistor $Q_{1}$ form the thermistor-controlled current source, producing the temperature-dependent voltage $V_{T}$ at the controller of $Q_{1}$. A low-pass filter $R_{3} C_{1}$ is incorporated to prevent the current source from oscillating. Voltage $V_{T}$ is then compared with the exponentially increasing voltage $V_{c}$ across $C_{c}$ by the comparator $A_{2}$. This comparison converts the linear change in $V_{T}$ into the exponential change over time. $V_{T}$ changes exponentially with $1 / T$, and the time required for $V_{c}$ to reach $V_{T}$ is inversely proportional to $T$. Therefore, repeating this comparison process by discharging $C$, one can obtain a linear relation between the oscillation frequency $f$ and temperature $T$.

The oscillation frequency of the $T / F$ converter using a thermistor with the material constant $B=1896$ and $R_{0}=$ $458 \Omega$ at $T_{0}=298 \mathrm{~K}$ is plotted in Fig. 5. From this plot, the offset frequency $f_{o}$ and the conversion sensitivity $S_{c}$ of the present $T / F$ converter are found to be $2695 \mathrm{~Hz}$ and

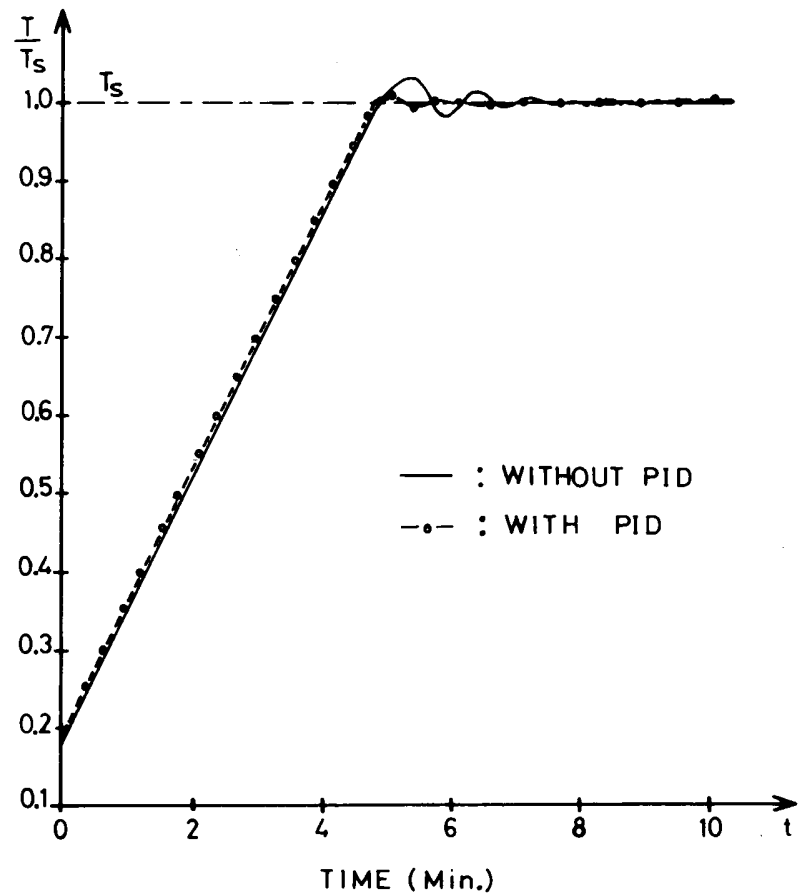

Fig. 6. The controller response to step change in the target temperature with and without the PID algorithm.

$30 \mathrm{~Hz} /{ }^{\circ} \mathrm{C}$, respectively. These values are stored in the control program to convert the target temperature into the frequency.

This prototype controller was applied to the temperature control of a water bath. Fig. 6 shows the controller response with and without the PID algorithm. Because of the small damping factor of the PLL, the step response without the PID control (solid line) oscillates around the target temperature. The PID algorithm for suppressing the oscillation is executed using the sampled data and thus the PID parameters should be determined from the PLL response in the $z$ domain [7]. In this system, however, the discrete response was approximated by a continuous one because the thermal time constant $C R$ of the water bath is much larger than the sampling period, and the conventional approach using the step response [8] was adopted to find the optimum PID parameters $K_{R}=17.73, K_{I}=$ 8.16, and $K_{D}=0.231$. Experimental data in Fig. 6 demonstrate the performance of the controller using the PID algorithm. Temperature of the water bath tracks to the target temperature closely and the overshoot and undershoot are greatly reduced. The error in the steady-state is within $0.1^{\circ} \mathrm{C}$.

\section{Conclusions}

A new technique for temperature control based on the PLL has been proposed and confirmed experimentally by the prototype controller. The principles of control are compatible with intelligent sensors now being developed and are applicable to the control of other quantities such as humidity, pressure, and flow.

The prototype controlier presented here features low cost, high precision, and programmability, but its range is limited to $100^{\circ} \mathrm{C}$ by the $T / F$ converter. To expand the 
control range, a $T / F$ converter using a platinum resistance thermometer is now under development.

\section{REFERENCES}

[1] Constant temperature baths and circulators. Neslab Instruments, Inc. Portsmouth, NH, 1980.

[2] M. Kaucsar, "Digitally programmable temperature programmer,", Elec. Eng., Mar. 1980.

[3] R. J. Muha, S. M. Gates, and J. T. Yates, Jr., "Digital temperature programmer for isothermal and thermal desorption measurements," Rev. Sci. Instr., vol. 56, pp. 613-614, 1985.
[4] Jeng-Renyang and Juht Zeng Lue, "A microcomputer-based programmable temperature controller," IEEE Trans. Instrum. Meas., vol. IM36, pp. 87-91, Mar. 1987.

[5] S. Middelhoek, P. J. French, J. H. Huijising, and W. J. Lian, "Sensors with digital or frequency output," in Proc. Transducers ' $87, \mathrm{pp}$. 17-24, 1987.

[6] Osama I. Mohamed, Toshifumi Takaoka, and K. Watanabe, "A simple linear temperature-to-frequency converter using a thermistor," Trans. IEICE, vol. E-70, Aug. 1987.

[7] C. L. Phillips and H. T. Nagle, Jr, Digital Control System Analysis and Design. Englewood Cliffs, NJ: Prentice-Hall, 1984.

[8] J. K. Astrom, “Adaptive feedback control,"' in Proc. IEEE, vol. 75, pp. 185-217, Feb. 1987. 